\title{
LINC00152 is a potential biomarker involved in the modulation of biological characteristics of residual colorectal cancer cells following chemoradiotherapy
}

\author{
ZHENGTING CHEN $^{1 *}$, XINYI CAI ${ }^{2 *}$, LI CHANG $^{1}$, YAOXIONG XIA $^{1}$, LI WANG $^{1}$, YU HOU $^{1}$, LAN LI $^{1}$, \\ DINGGUO PAN ${ }^{2}$, FURONG LI ${ }^{2}$, SHAN LIU ${ }^{3}$, WEI XIONG ${ }^{1}$ and WENHUI LI ${ }^{1}$ \\ Departments of ${ }^{1}$ Radiation Oncology, ${ }^{2}$ Colorectal Surgery and ${ }^{3}$ Combined Traditional Chinese and Western Medicine, \\ Yunnan Cancer Hospital, The Third Affiliated Hospital of Kunming Medical University, Kunming, Yunnan 650100, P.R. China
}

Received April 25, 2017; Accepted November 22, 2017

DOI: $10.3892 / \mathrm{ol} .2018 .7833$

\begin{abstract}
Concurrent radiotherapy and chemotherapy is a widely used, comprehensive treatment for rectal cancer. By studying the impact of concurrent chemoradiotherapy on the invasion and migration of colorectal cancer (CRC) cells and researching the associated molecular mechanisms, the present study aimed to provide a novel method to improve the therapeutic effect of this treatment against CRC. Human HCT116 and HT29 CRC cells were simultaneously treated with $4 \mathrm{~Gy}$ of $6 \mathrm{MV}$ X-rays and $10 \mu \mathrm{mol} / 15$-fluorouracil to establish a residual cell model. Transwell migration and invasion experiments were used to analyse the invasion and migration of the cells. The expression of long non-coding (lnc)RNAs was detected using a gene chip, and reverse transcription-quantitative polymerase chain reaction analysis was used to determine lncRNA expression levels. Specific small interfering RNAs were transfected into HCT116 residual cells to silence the expression of the identified key genes. The migration and invasion of residual CRC cells were demonstrated to be significantly increased compared with the original cells. Pvtl oncogene, long-chain non-protein-coding RNA 152 (LINC00152), and MIR22 host gene were selected as potential targets. However, the migration and invasion of residual HCT116 cancer cells were only significantly decreased following silencing of LINC00152 expression. LINC00152 may therefore be a potential biomarker
\end{abstract}

Correspondence to: Professor Wenhui Li or Dr Wei Xiong, Department of Radiation Oncology, Yunnan Cancer Hospital, The Third Affiliated Hospital of Kunming Medical University, 519 Kunzhou Road, Kunming, Yunnan 650100, P.R. China

E-mail: wenhuili64@yeah.net

E-mail:kmxwpaper@163.com

${ }^{*}$ Contributed equally

Key words: colorectal cancer cells, radiotherapy, chemotherapy, invasion, metastasis involved in modulation of the biological characteristics of residual CRC cells following chemoradiotherapy.

\section{Introduction}

The global incidence and mortality rates of colorectal cancer (CRC) are high. In 2012, the estimated global incidence of novel CRC cases diagnosed was 1.4 million, with 693,900 mortalities, and the number of novel cases and mortalities is continuously increasing due to economic developments and lifestyle changes in developing countries (1). Rectal cancer accounts for a large proportion of CRC cases and is accountable for $28 \%$ of CRC cases in America (2). The standard treatment for locally advanced rectal cancer is neoadjuvant chemoradiotherapy with radical surgery, and this treatment pattern may help achieve a complete pathological response (pCR) (3). Thus, the rates of surgical resection and anal retention are increasing. However, treatments for rectal cancer remain far from satisfactory due to the occurrence of post-treatment cancer recurrence and metastasis.

The phenomenon of radiotherapy or chemotherapy altering invasion and metastasis in tumor cells has begun to attract increased attention. Certain studies have demonstrated that treatment with chemotherapy drugs and radiotherapy may result in the opposite to the desired effect in patients with tumors; ignoring the potential additional side effects (4-6). However, the details of this phenomenon in CRC remain uncertain. Certain studies have verified that radiotherapy may result in CRC cell adhesion to the vascular endothelium, and radiotherapy may cause increased extracellular matrix deposition and the expression of matrix metalloproteinases (MMPs) in CRC cells $(7,8)$. As a result, the invasive potential of residual tumor cells may be increased following ionizing radiation treatment. Other studies have reported opposing results (9). Furthermore, the effects of radiotherapy on the invasion and metastasis of tumor cells vary with radiation therapy style and the tumor cell type. In addition, the effects of radiation on the expression of CRC-dependent proteins have been reported to be time- and dose-dependent $(10,11)$.

The prognosis of patients with locally advanced rectal cancer who underwent neoadjuvant radiotherapy and 
chemotherapy and achieved a pCR was revealed to be significantly improved compared with patients who did not achieve a pCR (12). However, the rate of pCR following neoadjuvant chemoradiotherapy is only $\sim 10 \%$ (13). Therefore, the majority of these patients do not achieve a pCR, meaning that residual tumor cells are present. These patients must then wait 6-8 weeks for surgery. During this period, the invasive and metastatic potentials of residual rectal tumor cells may have increased, thus affecting the overall therapeutic effect in patients with rectal cancer.

The key mechanisms underlying this change remain uncertain. Long non-coding RNAs (lncRNAs) are non-coding RNA molecules (>200 nt) that affect corresponding metabolic processes or directly affect mRNA expression (14). LncRNAs have previously been demonstrated to be associated with invasion, metastasis and prognosis in CRC (15). Therefore, certain lncRNAs may account for the biological changes in the characteristics of residual CRC cells following chemoradiotherapy. Further studies are required to explore these changes and associated mechanisms, and this was the goal of the present study.

\section{Materials and methods}

Cell cultures. The human CRC HCT116 and HT29 cell lines were purchased from the Kunming Institute of Zoology, Chinese Academy of Sciences (Kunming, China). Cells were cultured in RPMI-1640 medium (Hyclone; GE Healthcare Life Sciences, Logan, UT, USA) with $10 \%$ fetal bovine serum (FBS; Gibco, Thermo Fisher Scientific, Inc., Waltham, MA, USA) and $1 \%$ penicillin and streptomycin. Cells were cultured in an incubator at $37^{\circ} \mathrm{C}$ in an atmosphere containing $5 \% \mathrm{CO}_{2}$.

Concurrent chemoradiation of CRC cells in vitro. The chemoradiation model was established by culturing cells in RPMI-1640 medium (at $37^{\circ} \mathrm{C}$ ) treated with $10 \mu \mathrm{mol} / 1$ 5-fluorouracil (5-FU) (Shanghai Aladdin Bio-Chem Technology Co., Ltd., Shanghai, China) for $24 \mathrm{~h}$ and simultaneously exposing them to 4 Gy of $6 \mathrm{MV}$ X-ray irradiation (PrecisePlan A-C, iView-GT; Elekta Instrument AB, Stockholm, Sweden) when the cells had grown in cell culture flask to between 80 and $100 \%$ confluence, as previously described (16). The chemical and irradiation treatments were repeated once the cells had reached $>80 \%$ confluence. A residual CRC cell model was established following 4 treatment rounds in the HCT116 and HT29 cells.

Migration and invasion assays using original and residual cells. According to the methods described in a previous study (17), a Transwell assay was used to study cell migration and invasion. For the migration study, $200 \mu \mathrm{l}$ single cells suspension $\left(2 \times 10^{4}\right.$ cells/ chamber) with serum-free RPMI-1640 medium was added to the upper chamber, and $600 \mu$ l RPMI-1640 medium with 10\% FBS was added to the lower chamber in each well of a 24 -well plate. Then, the Transwell chambers (24-well, $8-\mu \mathrm{m}$ pore size; EMD Millipore, Billerica, MA, USA) and the 24-well plates were incubated at $37^{\circ} \mathrm{C}$ in a $5 \% \mathrm{CO}_{2}$ atmosphere. After $48 \mathrm{~h}$, the plate was removed from the incubator and a wet cotton swab was used to remove cells from the top of the upper chamber. Next, the remaining cells were fixed for analysis using pure methanol for $30 \mathrm{~min}$ at room temperature, and then stained with $0.1 \%$ crystal violet for $15 \mathrm{~min}$ at room temperature. Then, cells in at least 5 fields of view were counted under an inverted microscope, and images were captured using the Leica 3000 Suite Application (version 3.8.0; Leica Microsystems GmbH, Wetzlar, Germany).

For the invasion study, the Transwell inserts were first covered with $80 \mu \mathrm{l}$ Matrigel (200-300 $\mu \mathrm{g} / \mathrm{ml}$, Corning Incorporated, Corning, NY, USA). Then, $10 \times 10^{4}$ cells were seeded in the upper chamber. The subsequent steps were the same as those for the migration assay.

IncRNA expression profile detection. The original and residual cancer cells derived from the HCT116 cell line (HCT116N and HCT116CR, respectively), were routinely digested with $0.25 \%$ trypsin-EDTA (Invitrogen; Thermo Fisher Scientific, Inc.) and centrifuged at $100 \mathrm{x}$ g for $5 \mathrm{~min}$ at room temperature. The cells were subsequently collected, treated with TRIzol (Invitrogen; Thermo Fisher Scientific, Inc.) according to the manufacturer's protocol, and sent to the biological company KangChen Bio-tech, Inc. (Shanghai, China) for lncRNA expression profile detection with the Human LncRNA Array v3.0 (8x60 K; Arraystar, Inc., Rockville, MD, USA). Differentially expressed lncRNAs were screened with a threshold of fold-change $>2$ and $\mathrm{P}<0.05$. In addition, Gene Ontology $(18,19)$ and Kyoto Encyclopedia of Genes and Genomes (20-22) analyses were used to classify the differentially expressed lncRNAs and analyze the associated signaling pathways with false discovery rate $<1 \%$.

Reverse transcription-quantitative polymerase chain reaction (RT-qPCR). RT-qPCR was performed to detect the expression of MIR22 host gene (MIR22HG), long-chain non-protein-coding RNA 152 (LINC00152) and Pvt1 oncogene (PVT1) in cells. RT-qPCR was also used to identify the effects of silencing the expression of these three lncRNAs in HCT116CR cells. The primer sequences are listed in Table I. $\beta$-Actin was used as the internal control. All the following operations were performed according to the manufacturer's protocol. TRIzol reagent was used to extract total RNA from cells. Total RNA was converted to cDNA at $37^{\circ} \mathrm{C}$ for $60 \mathrm{~min}$ by using Quantscript RT Kit (Tiangen Biotech Co., Ltd., Beijing, China) according to the manufacturer's protocol. Each sample had three replicates, was amplified in a $10-\mu 1$ reaction mixture (containing $1 \mu \mathrm{lcDNA}$ and $0.6 \mu \mathrm{l}$ primer) by applying FS Universal SYBR Green Master (Roche Diagnostics, Basel, Switzerland). The thermocycling conditions were: initial denaturation at $95^{\circ} \mathrm{C}$ for $10 \mathrm{~min}$, followed by 45 cycles of; $95^{\circ} \mathrm{C}$ for $15 \mathrm{sec}$ and $60^{\circ} \mathrm{C}$ for $60 \mathrm{sec}$. Samples were analyzed using an ABI QuantStudio 6 Flex system (Applied Biosystems; Thermo Fisher Scientific). The relative gene expression levels in cells were calculated using the $2^{-\Delta \Delta C q}$ method (23).

siRNA transfection of HCT116CR cells. Specific small interfering (si)RNAs against the selected genes were provided by Shanghai GenePharma Co., Ltd. (Shanghai, China). The siRNA sequences are listed in Table II. HCT116CR cells in the logarithmic growth phase were seeded in 24-well plates at a density of $5 \times 10^{4}$ cells per well. The culture medium consisted of $500 \mu \mathrm{l}$ antibiotic-free Opti-minimum essential medium (MEM; Invitrogen; Thermo Fisher Scientific, Inc.) containing 
Table I. Primer sequences for PVT1, LINC00152 and MIR22HG.

\begin{tabular}{ll}
\hline Name & \multicolumn{1}{c}{ Primer sequences (5'-3') } \\
\hline$\beta$ - $\alpha$ ctin-F & AGCACAGAGCCTCGCCTTTG \\
$\beta$ - $\alpha$ ctin-R & CTTCTGACCCATGCCCACCA \\
PVT1-F & GAGAGAATCCTGTTACACCTGGG \\
PVT1-R & CGACCTGGTTTCTCGTGAGC \\
LINC00152-F & ACAAGCGGTGCCTGAGCC \\
LINC00152-R & CCGACTCTCCTACACATCCACAG \\
MIR22HG-F & TGGGAAGGTCCGAACAGCA \\
MIR22HG-R & GGGAGAATTTCCTGTCTGCACA
\end{tabular}

F, forward; R, reverse; PVT1,Pvt1 oncogene; LINC00152, long-chain non-protein-coding RNA 152; MIR22HG, MIR22 host gene.

Table II. SiRNA sequences against PVT1, LINC00152 and MIR22HG.

\begin{tabular}{ll}
\hline siRNA & \multicolumn{1}{c}{ Sequence (5'-3') } \\
\hline PVT1-444-s & GCUUCAAGCUCACGAGAAATT \\
PVT1-444-as & UUUCUCGUGAGCUUGAAGCTT \\
PVT1-368-s & GGACUUGAGAACUGUCCUUTT \\
PVT1-368-as & AAGGACAGUUCUCAAGUCCTT \\
PVT1-302-s & CCUGUUACACCUGGGAUUUTT \\
PVT1-302-as & AAAUCCCAGGUGUAACAGGTT \\
LINC00152-481-s & GCAGAAGACAAAGCCGAAATT \\
LINC00152-481-as & UUUCGGCUUUGUCUUCUGCTT \\
LINC00152-738-s & GCAUGAUUGGAUGAUGUUTT \\
LINC00152-738-as & AAACAUCAUCCAAUCAUGCTT \\
LINC00152-619-s & GGGAGACAGUUCACAGAUATT \\
LINC00152-619-as & UAUCUGUGAACUGUCUCCCTT \\
MIR22HG-111-s & CCCUGGGAACAAGUCAGUUTT \\
MIR22HG-111-as & AACUGACUUGUUCCCAGGGTT \\
MIR22HG-457-s & GAAGGCUCAAACAACCCAATT \\
MIR22HG-457-as & UUGGGUUGUUUGAGCCUUCTT \\
MIR22HG-470-s & ACCCAAGGUGGUAUGUGAUTT \\
MIR22HG-470-as & AUCACAUACCACCUUGGGUTT \\
Negative control-s & UUCUCCGAACGUGUCACGUTT \\
Negative control-as & ACGUGACACGUUCGGAGAATT
\end{tabular}

siRNA, small interfering RNA; s, sense; as, antisense; PVT1, Pvt1 oncogene; LINC00152, long-chain non-protein-coding RNA 152; MIR22HG, MIR22 host gene.

$10 \%$ FBS. Cells were cultured in a $\mathrm{CO}_{2}$ incubator for $24 \mathrm{~h}$ until reaching $\sim 70 \%$ confluence. Then, $1 \mu 1$ Lipofectamine 2000 (Invitrogen; Thermo Fisher Scientific, Inc.) was diluted with $50 \mu \mathrm{l}$ Opti-MEM, and 20 pmol siRNA was diluted with $50 \mu 1$ Opti-MEM. The diluted Lipofectamine reagent and siRNA were then mixed, and then the siRNA and lipofectamine mixture $(100 \mu \mathrm{l})$ was added to each well of the prepared cells. Next, the cells were incubated $\left(37^{\circ} \mathrm{C}, 5 \% \mathrm{CO}_{2}\right)$ for $6 \mathrm{~h}$. The culture medium was then replaced with normal RPMI-1640 medium with $10 \% \mathrm{FBS}$, and the cells were cultured for an additional $48 \mathrm{~h}$ in the same incubator. The cells were then collected for use in subsequent steps.

Migration and invasion assays of HCT116CR and siRNA-transfected HCR116CR cells. Transwell assays were used to study the migration of HCT116CR and siRNA-transfected HCT116CR cells. Cells were collected and resuspended in serum-free RPMI-1640 medium. In the lower chamber, $600 \mu 1$ medium containing 10\% FBS was added. Each prepared cell suspension $\left(150 \mu 1 ; 3 \times 10^{4}\right.$ cells/chamber) was added to the upper chambers, and the cells were incubated at $37^{\circ} \mathrm{C}$ and $5 \% \mathrm{CO}_{2}$ in an incubator for $24 \mathrm{~h}$. The chambers were then removed, and the fluid was aspirated. Then, the cells were fixed with pure methanol $(800 \mu \mathrm{l} / \mathrm{ch}$ amber) for $30 \mathrm{~min}$ and stained with diluted Giemsa Stain solution (10xStock solution; Beijing Solarbio Science \& Technology Co., Ltd., Beijing, China) for $20 \mathrm{~min}$. A wet cotton swab was used to wipe the cells off the bottom of the upper chamber. Tweezers were used to peel off the membrane and turn it upside down to dry. The dried membrane was then transferred to a glass slide and mounted with a piece of neutral gum. Finally, the cells were counted in at least 5 fields of view and images were captured under a light microscope.

For the invasion assays, Matrigel was diluted to a final concentration of $1 \mathrm{mg} / \mathrm{ml}$ with $4^{\circ} \mathrm{C}$ precooled serum-free RPMI-1640 medium. A volume of $100 \mu \mathrm{l}$ diluted Matrigel was added vertically to the bottom of the upper chamber and incubated at $37^{\circ} \mathrm{C}$ for $4-5 \mathrm{~h}$ to dry. The next steps were the same as those aforementioned for the migration assay.

Statistical analysis. Where the data are normally distributed, they are reported as the mean \pm standard deviation. Non-normally distributed data is presented as the median. Statistical analyses were performed using SPSS 20.0 software (IBM Corp., Armonk, NY, USA). Unpaired Student's t tests were used for comparison between two groups, and one-way analysis of variance followed by Dunnett's tests was used for comparisons between three or more groups. All experiments were repeated at least three times. $\mathrm{P}<0.05$ was considered to indicate a statistically significant difference.

\section{Results}

Residual HCT116 and HT29 CRC cell models. HCT116 and HT29 CRC cells were simultaneously treated with $10 \mu \mathrm{mol} / 1$ 5-FU and irradiation (4 Gy 6 MV X-ray). The cells consistently died from the fourth day to the tenth day following treatment. Then, the remaining cells slowly proliferated until reaching confluence. Morphological changes in the residual CRC cells were determined. For example, a number of large cells were observed, with volumes three or four times greater than the normal cells, and the new cells growing around the large cells exhibited diverse pseudopodia. In addition, larger intercellular gaps were observed by light microscopy (Fig. 1). These residual CRC cells were designated HCT116CR and HT29CR cells.

Residual CRC cells demonstrated increased migration and invasion in vitro. Once the residual CRC cell model was 


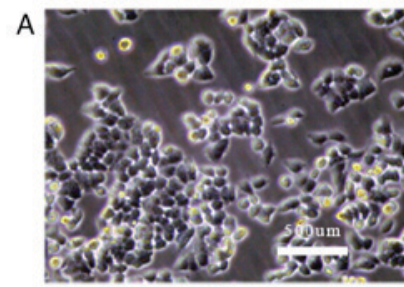

HCT116N

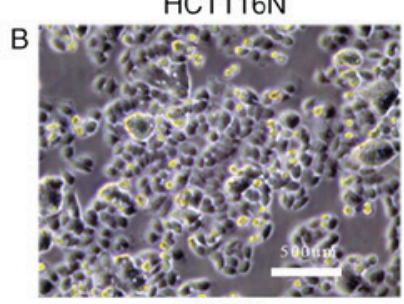

HT29N

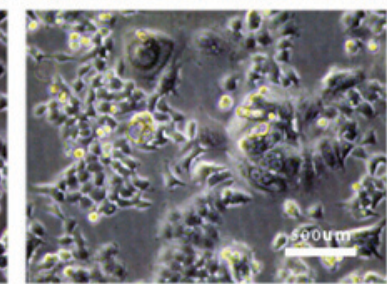

HCT116CR

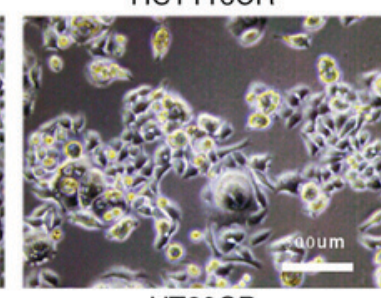

HT29CR
Figure 1. Morphological changes in residual CRC cells following chemoradiation therapy. (A) HCT116 cells and (B) HT29 cells were examined. The intercellular gaps between residual CRC cells increased, the shapes of residual cells were varied, and the residual cells exhibited pseudopodia. $\mathrm{CRC}$, colorectal cancer; $\mathrm{N}$, original normal cell; $\mathrm{CR}$, residual cells following chemoradiation.

established, the migration and invasion of the original and residual cells were compared (Fig. 2). A significantly increased number of residual cells migrated compared with original cells; 15.33 \pm 5.07 HCT116N cells migrated, while 46.56 \pm 7.97 HCT116CR cells migrated ( $\mathrm{P}=0.005$; Fig. $2 \mathrm{~A}$ and $\mathrm{C})$. A similar trend was observed for the HT29 cells, as 15.83 \pm 9.88 original cells and $59.16 \pm 20.73$ residual cells migrated $(\mathrm{P}=0.003$; Fig. 2A and C). The invasion assay also revealed that more residual cells passed through the membrane, as $59.17 \pm 13.34$ HCT116N cells invaded and 149.63 \pm 7.65 HCT116CR cells invaded ( $\mathrm{P}<0.001$; Fig. 2B and D). In addition, 35.97 \pm 2.89 and 96.00 \pm 8.13 HT29N and HT29CR cells invaded, respectively $(\mathrm{P}<0.001$; Fig. 2B and D).

HCT116CR and HCT116N cell lncRNA expression profiling. Having identified differences in the migratory and invasive potentials of HCT116N and HCT116CR cells, IncRNA expression levels in HCT116N and HCT116CR cells were then detected and analyzed. A total of 18,928 lncRNAs were detected by biochip, 2,662 of which were differentially expressed. Among these, 1,245 lncRNAs were upregulated and 1,417 $\mathrm{lncRNAs}$ were downregulated. Three lncRNAs, PVT1, LINC00152 and MIR22HG, which were upregulated in HCT116CR cells compared with HCT116N cells were selected according to associated reviews and analyses (24-26). The expression levels of these three selected lncRNAs in the cell types were confirmed by RT-qPCR, with the PCR results being consistent with the biochip results.

Results of siRNA silencing as detected by RT-qPCR. To investigate the role of the three lncRNAs (PVT1, LINC00152 and MIR22HG) in residual CRC cells, HCT116CR cells were transfected with siRNAs against the three selected lncRNAs (PVT1, LINC00152 and MIR22HG). The results of siRNA transfection were assessed by RT-qPCR. The most effective specific siRNAs for each molecular target were PVT1-368, LINC00152-481 and MIR22HG-111. The expression levels of
PVT1, LINC00152 and MIR22HG in HCT116CR cells were significantly decreased compared with the control group, with the relative expression level of each target gene decreased by 58,55 and $61 \%$, respectively $(\mathrm{P}<0.01$; Fig. 3$)$.

Residual CRC cell biological characteristics are modulated via LINC00152 silencing. To compare the effects of siRNA transfection on the migration and invasion of HCT116 residual cancer cells, the Transwell experiments were performed again (Fig. 4). In the migration experiment, a total of $206.00 \pm 29.46$ cells passed through the chamber membrane in the negative control (NC) group, $186.00 \pm 21.63$ cells in the MIR22HG-siRNA group ( $\mathrm{P}=0.618$ compared with the $\mathrm{NC}$ group), 65.00 \pm 24.06 cells in the LINC00152-siRNA group $(\mathrm{P}<0.001$ compared with the $\mathrm{NC}$ group) and 194.33 \pm 17.21 in the PVT1-siRNA group $(\mathrm{P}=0.875$ compared with the NC group; Fig. 4A and C). Only the number of cells in the LINC00152-siRNA group was significantly reduced, suggesting a significant decline in the mobility of HCT116CR cells transfected with LINC00152 siRNA.

Similar to the migration experiment, a significant difference in invasion was only observed in the LINC00152-siRNA group compared with the NC group. The number of invading cells in the control group was $57.00 \pm 12.53$, while the numbers in the LINC00152-siRNA, MIR22HG-siRNA and PVT1-siRNA groups were 9.67 $44.73(\mathrm{P}=0.005$ compared with the NC group), 45.67 \pm 10.02 ( $\mathrm{P}=0.590$ compared with the $\mathrm{NC}$ group) and $41.00 \pm 19.31(\mathrm{P}=0.347$ compared with the NC group), respectively (Fig. $4 \mathrm{~B}$ and D). There was no significant difference between number of invading cells in the MIR22HG-siRNA or PVT1-siRNA groups and the control group.

\section{Discussion}

In the cell experiments of the present study, residual CRC cell models were successfully established via repeated concurrent chemoradiotherapy, which was intended to mimic the clinical treatment model as closely as possible. The morphological changes observed in residual CRC cells following chemoradiation therapy indicated that the biological characteristics may also be altered. In addition, Transwell experiments demonstrated that the migration and invasion of the residual cell lines were significantly increased compared with the original cells. These results agree with the results of several previous studies (7,27-29). A similar phenomenon has also been observed in multiple types of cancer cell. Yamauchi et al (6) demonstrated that the invasion and metastasis of human HT1080 fibrosarcoma cells was increased in cancer cell-bearing host mice pretreated with cyclophosphamide. Therefore, in addition to from side effects, chemotherapy drugs may exert effects opposite to those which are desired. Jadhav et al (4) irradiated human SK-N-AS neuroblastoma cells with different irradiation doses, and observed that irradiated cells had increased expression levels of urokinase-type plasminogen activator, MMP-9 and vascular endothelial growth factor compared with non-irradiated cells, as well as increased capillary-like structure of microvascular endothelial cells.

$\mathrm{X}$-rays were used to treat the CRC cells in the present study, and migration and invasion were increased in residual 
A
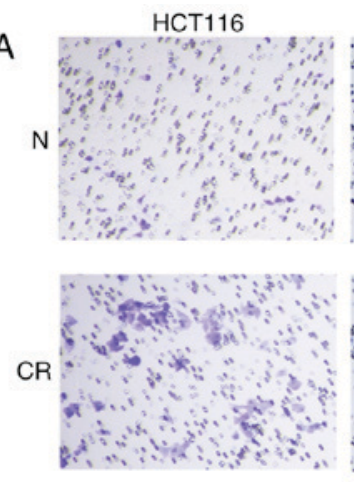

C

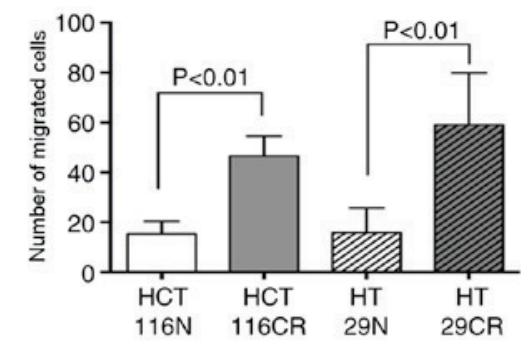

B
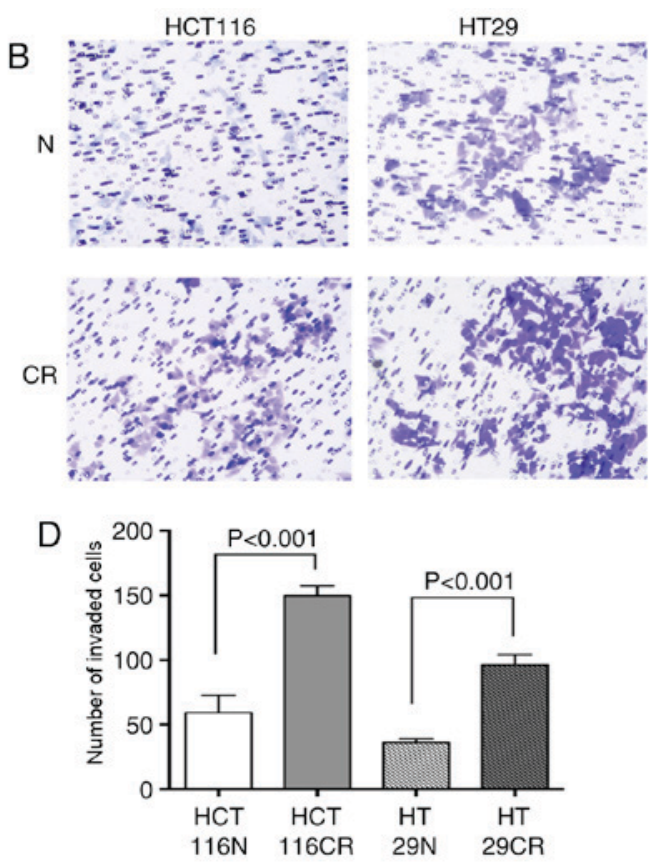

Figure 2. Migration and invasion increased in residual colorectal cancer cells following chemoradiation. Representative images (magnification, x100) from (A) the cell migration assay and (B) the cell invasion assay. The number of (C) migrated and (D) invaded cells per field.

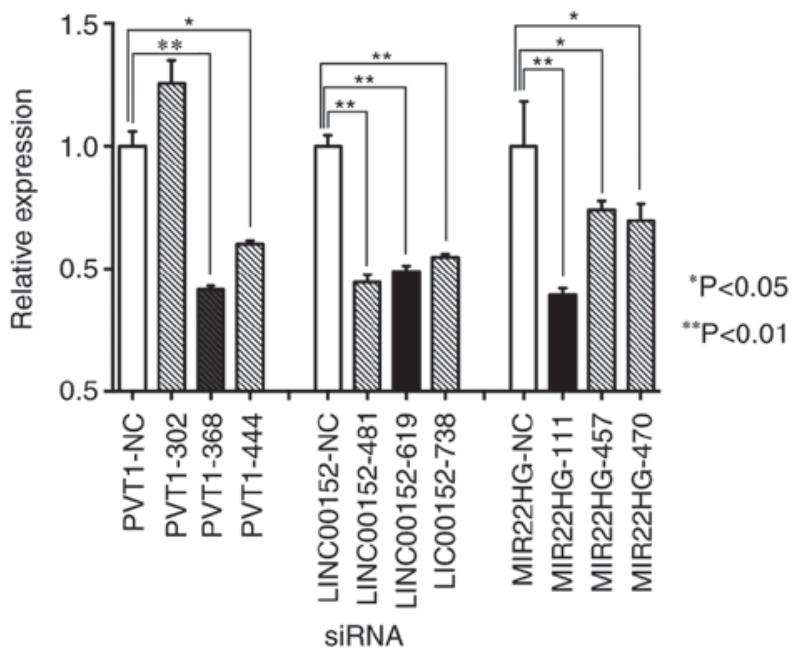

Figure 3. Reverse transcription-quantitative polymerase chain reaction analysis was used to analyze the result of transfection with siRNAs against PVT1, LINC00152 and MIR22HG in HCT116CR cells. Each selected gene had three siRNAs, and PVT1-302, LINC00152-481 and MIR22HG-111 most effectively silenced the expression of the corresponding lncRNAs. "P<0.05 and ${ }^{* *} \mathrm{P}<0.01$, with comparisons indicated by lines. HCT116CR, residual HCT116 cells following chemoradiation; PVT1, Pvt1 oncogene; LINC00152, long-chain non-protein-coding RNA 152; MIR22HG, MIR22 host gene; siRNA, small interfering RNA.

cells compared with original cells in the two CRC cell lines that were assessed, HCT116 and HT29. However, the use of X-ray irradiation to treat HCT116 cells has also been demonstrated to decrease invasion and metastasis of residual cancer cells via upregulation of KiSS-1 metastasis suppressor expression (30), which disagrees with the results of the present study. However, this previous study only observed the effect of radiotherapy and did not fully simulate concurrent clinical radiotherapy and chemotherapy. Therefore, further studies are required. The results of other previous studies suggest that the effects of radiotherapy on tumor cell invasion and metastasis may vary depending on the radiation mode and tumor cell type, and that the effect of radiotherapy on the migration of CRC cells is time- and dose-dependent $(10,11,31)$. Therefore, studies investigating alterations in the invasion and metastasis of residual cells following chemotherapy may need to assess various irradiation patterns, doses, observation times and types of CRC cell.

In addition, the mechanisms underlying these phenomena remain uncertain. The epithelial-to-mesenchymal transition (EMT) has been observed in CRC cells and rectal cancer tissues following neoadjuvant radiotherapy and chemotherapy, indicating that the invasion of residual rectal cancer cells was increased $(27,29)$. The morphological changes of the residual CRC cancer cells observed in the present study suggest that EMT occurred. In addition, a previous study demonstrated that invasion and metastasis were increased in residual CRC cells due to an increase in EPH receptor A4 expression levels and the extent of the EMT in these cells (28). There is also another view regarding the mechanism underlying the biological changes observed in residual CRC cells following radiotherapy. The degradation and destruction of the extracellular matrix and the basement membrane are important processes in tumor metastasis. An in vitro study revealed that radiotherapy treatment resulted in upregulated MMP expression in CRC cells (8). The increased expression of MMPs in CRC cells following radiotherapy may indicate an increase in cell invasion and metastasis. However, this change was transient, not continuous (8).

To explore the key mechanisms underlying the biological changes in residual CRC cells, the differential expression of IncRNAs was investigated. Among the three selected IncRNAs that were differentially expressed in residual CRC cells compared with original CRC cells, that may have been 

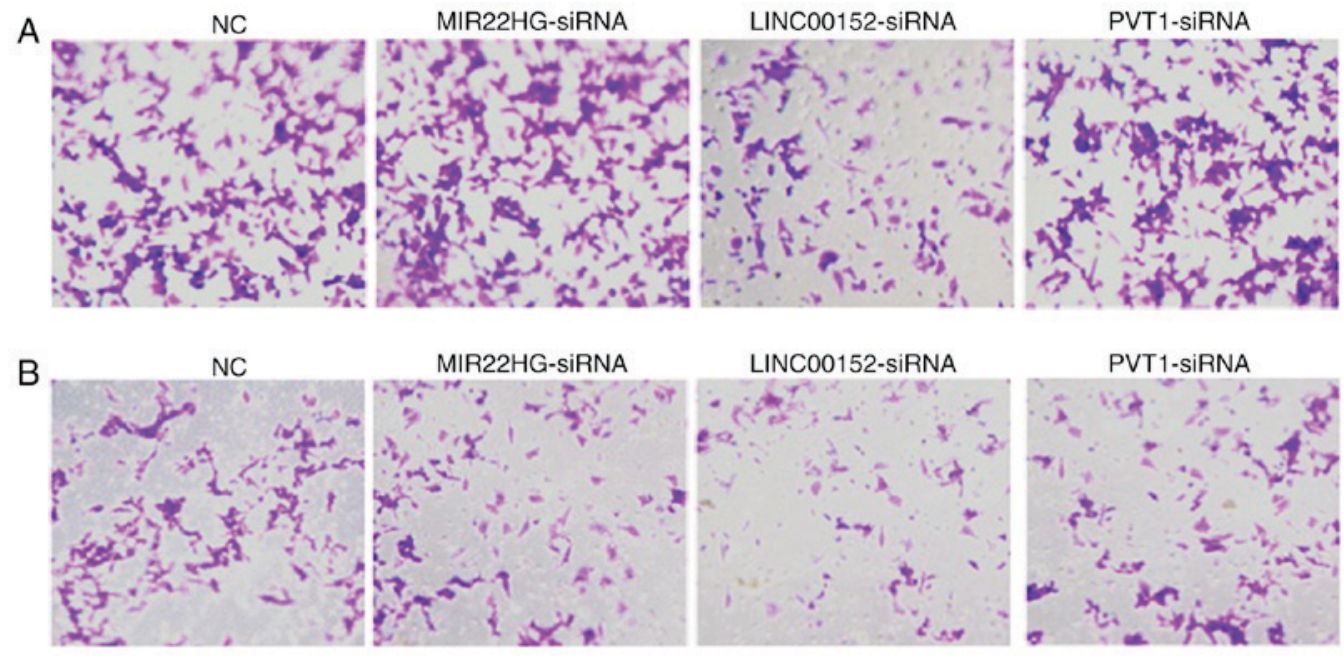

MIR22HG-siRNA

LINC00152-siRNA
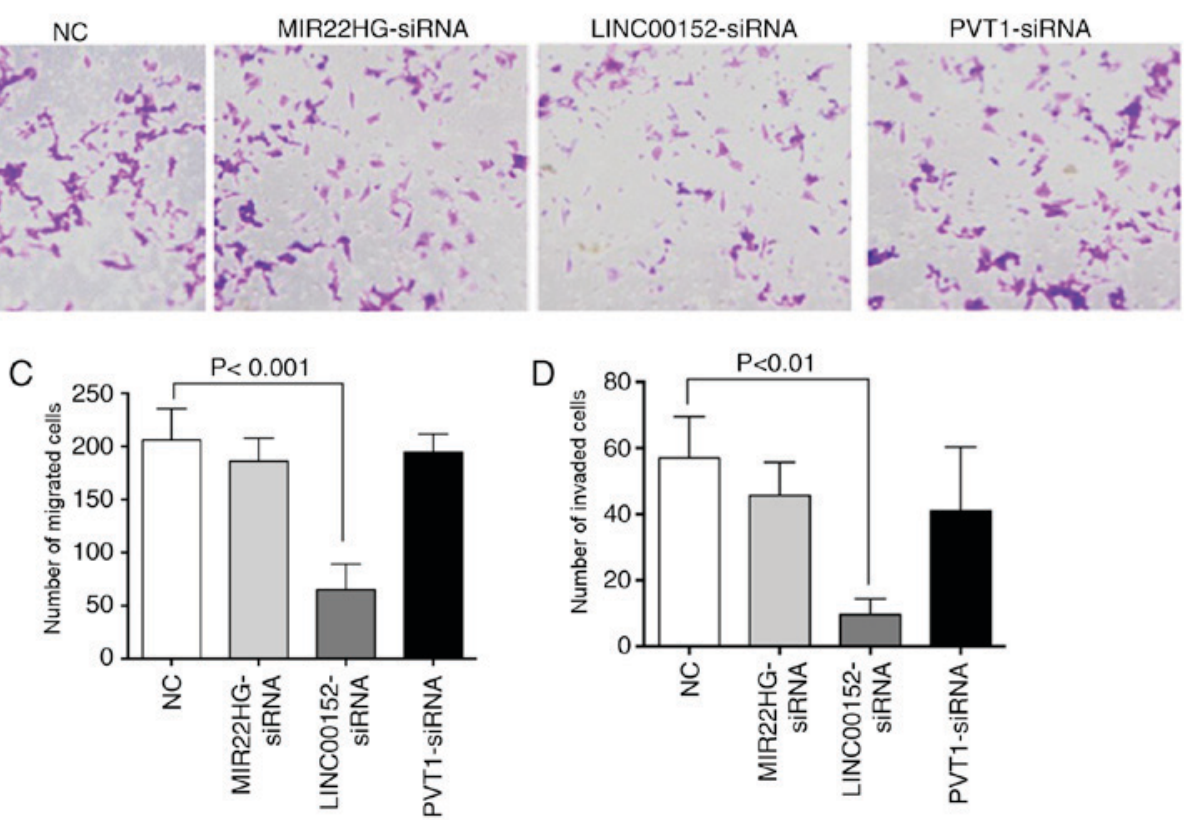

Figure 4. Transwell assays were used to compare migration and invasion in siRNA-transfected HCT116CR cells and HCT116CR cells. Representative images (magnification, $x 100$ ) of (A) cell migration and (B) cell invasion. The number of (C) migratory cells and (D) invading cells per field. siRNA, small interfering RNA; NC, negative control; PVT1, Pvt1 oncogene; LINC00152, long-chain non-protein-coding RNA 152; MIR22HG, MIR22 host gene; siRNA, small interfering RNA.

potential therapeutic molecular targets, only LINC00152 appeared to alter the biological characteristics of residual CRC cells. The invasive and metastatic potentials of residual CRC cells were decreased by silencing the expression of LINC00152.

LINC00152, which is known to regulate the cytoskeleton, may also be involved in cell cycle regulation and DNA damage repair. LINC00152 is a potential oncogene which may be involved in various types of cancer (32). In gastric cancer (GC), downregulation of LINC00152 expression decreased migration and invasion and suppressed proliferation and EMT progression in gastric cancer cells (33). In addition, LINC00152 has potential as a prognostic biomarker and therapeutic target in tongue squamous cell carcinoma, renal cell carcinoma, lung cancer, and gallbladder cancer (GBC) (34-37). In the present study, residual CRC cell migration and invasion were demonstrated to be significantly modulated by LINC00152 interference. In CRC, increased expression of LINC00152 is associated with clinical stage and lymph node metastasis, and may serve as a molecular marker for colon cancer metastasis (38). Furthermore, LINC00152 has been revealed to be associated with oxaliplatin (L-OHP) resistance in vitro and in vivo, and LIN00152 contributes to L-OHP resistance by acting as a competing endogenous RNA to regulate microRNA (miR)-193a-3p and erb-b2 receptor tyrosine kinase 4 expression (39). In other types of cancer, including hepatocellular carcinoma, LINC00152 activates the mechanistic target of rapamycin signaling pathway to increase tumorigenesis (40). In GC and lung adenocarcinoma (LAC), LINC00152 interacts with enhancer of zeste homologue 2, silencing the expression of $p 15$ and $p 21$ in GC and inhibiting interleukin 24 transcription in LAC, resulting in acceleration of the cell cycle and cell proliferation $(24,41)$. LINC00152 has also been revealed to promote proliferation in GC through an epidermal growth factor receptor-dependent pathway. Other studies have demonstrated that LINC00152 may contribute to renal cell carcinoma progression by epigenetically repressing P16 expression and interacting with miR-205 (35). In addition, the LINC00152/miR-138/hypoxia-inducible factor- $1 \alpha$ pathway potentiates the progression of GBC (37). The results of another previous study suggested that SP1 transcription factor/LINC00152/phosphoinositide 3-kinase/protein kinase B may be a potential therapeutic target in GBC (42). Overall, LINC00152 overexpression seems to serve a critical function in cancer development, and the associated mechanisms vary among different types of cancer. However, the functional regulatory mechanisms of LINC00152 remain uncertain, and the exact underlying mechanism requires further exploration. While LINC00152 has been a major area of research in multiple types of cancer, to the best of our knowledge the association between LINC00152 and the biological characteristics of residual CRC cells had not been previously reported. 
The results of the present study provide additional scientific support for the clinical value of LINC00152 in treating CRC.

However, patients with CRC patients with high LINC00152 expression in tumor tissues have been reported to have an improved prognosis than those with low LINC00152 expression (43). Zhang et al (44) demonstrated that LINC00152 overexpression reduces $\mathrm{CRC}$ cell viability and increases apoptosis, and LINC00152 may be downregulated by miR-367c-3p in CRC tissues and cells, but this previous study did not examine changes in the invasion and migration of CRC cells. Thus, further CRC cell experiments in clinical contexts concerning LINC00152 are merited.

The other two potential molecular targets that were selected, MIR22HG and PVT1, were also differentially expressed between the residual and original CRC cells. However, the migratory and invasive abilities of residual HCT116 cells were not significantly different from those of residual cells when MIR22HG or PVT1 expression was silenced. MIR22HG is an indicator for chemical stress response, and functions as an oncogene in ovarian cancer $(25,45)$. MIR22HG is also one of the most upregulated tumor suppressor genes in CRC cells under microgravity (46). In CRC cell lines, transforming growth factor- $\beta$ expression and the apoptotic signaling pathway are activated when PVT1 expression is downregulated, the proliferation and invasion of CRC cells are reduced, and high PVT1 expression in patients with CRC is associated with a poor prognosis (26). In the present study, CRC cells treated with radiotherapy and chemotherapy had relatively high PVT1 and MIR22HG expression compared with untreated cells. However, there were no significant differences in the invasion and metastasis of residual CRC cells following PVT1 and MIR22HG silencing. These results indicated that neither PVT1 nor MIR22HG is a key diagnostic or therapeutic target in the process of biological characteristic alterations caused by radiotherapy and chemotherapy in CRC cells.

To the best of our knowledge, the present study is the first to examine the effects of radiotherapy and chemotherapy on the invasion and metastasis of CRC cells and reveal a relevant potential biomarker. These results may help improve the therapeutic effects of CRC treatments. However, there were two main limitations with the present study. First, only two cell lines and limited doses of one radiation therapy type were used. Second, this research was conducted entirely in vitro. Therefore, the aim of our future studies is to perform ethical animal experiments and clinical research regarding LINC00152 and alterations of the biological characteristics of residual CRC cells.

In conclusion, the invasion and metastasis of residual CRC cells increased following radiotherapy and chemotherapy, indicating that these biological characteristics of residual cancer cells were altered by this treatment. In addition, the lncRNA LINC00152 was revealed to be a potential biomarker that modulates the alterations caused by these treatments in CRC. The present study provides a robust scientific basis for further research to improve the therapeutic effects of CRC treatments.

\section{Acknowledgements}

The present study was supported by the National Natural Science Foundation of China (grant no. 81301825), the
Training Program for Medical Reserve Talents of the Health and Family Planning Commission of Yunnan Province (grant nos. H-201641, H-201624), and the National Key Clinical Specialty (Oncology) Fund.

\section{References}

1. Torre LA, Bray F, Siegel RL, Ferlay J, Lortet-Tieulent J and Jemal A: Global cancer statistics, 2012. CA Cancer J Clin 65: 87-108, 2015.

2. Siegel R, Desantis $\mathrm{C}$ and Jemal A: Colorectal cancer statistics, 2014. CA Cancer J Clin 64: 104-117, 2014.

3. Benson AB III, Venook AP, Bekaii-Saab T, Chan E, Chen YJ, Cooper HS, Engstrom PF, Enzinger PC, Fenton MJ, Fuchs CS, et al: Rectal cancer, version 2.2015. J Natl Compr Canc Netw 13: 719-728, 2015.

4. Jadhav U and Mohanam S: Response of neuroblastoma cells to ionizing radiation: Modulation of in vitro invasiveness and angiogenesis of human microvascular endothelial cells. Int J Oncol 29: 1525-1531, 2006

5. Xiong W, Ren ZG, Qiu SJ, Sun HC, Wang L, Liu BB, Li QS, Zhang W, Zhu XD, Liu L, et al: Residual hepatocellular carcinoma after oxaliplatin treatment has increased metastatic potential in a nude mouse model and is attenuated by Songyou Yin. BMC Cancer 10: 219, 2010.

6. Yamauchi K, Yang M, Hayashi K, Jiang P, Yamamoto N, Tsuchiya H, Tomita K, Moossa AR, Bouvet M and Hoffman RM: Induction of cancer metastasis by cyclophosphamide pretreatment of host mice: An opposite effect of chemotherapy. Cancer Res 68: 516-520, 2008.

7. Meineke V, Gilbertz KP, Schilperoort K, Cordes N, Sendler A, Moede $\mathrm{T}$ and van Beuningen D: Ionizing radiation modulates cell surface integrin expression and adhesion of COLO-320 cells to collagen and fibronectin in vitro. Strahlenther Onkol 178: 709-714, 2002.

8. Speake WJ, Dean RA, Kumar A, Morris TM, Scholefield JH and Watson SA: Radiation induced MMP expression from rectal cancer is short lived but contributes to in vitro invasion. Eur J Surg Oncol 31: 869-874, 2005.

9. Hasegawa T, Sato M, Kurimoto M, Takahashi H, Kawashima T, Matsuo Y, Yamamoto M, Sawai H, Funahashi H, Okada Y, et al: Biological effect of irradiation on adhesion molecules in human colon cancer cells in vitro. Anticancer Res 25: 875-879, 2005

10. Goetze K, Scholz M, Taucher-Scholz G and Mueller-Klieser W: The impact of conventional and heavy ion irradiation on tumor cell migration in vitro. Int J Radiat Biol 83: 889-896 2007.

11. Suetens A, Moreels M, Quintens R, Soors E, Buset J, Chiriotti S, Tabury K, Gregoire V and Baatout S: Dose- and time-dependent gene expression alterations in prostate and colon cancer cells after in vitro exposure to carbon ion and X-irradiation. J Radiat Res 56: 11-21, 2015.

12. Jalilian M, Davis S, Mohebbi M, Sugamaran B, Porter IW, Bell S, Warrier SK and Wale R: Pathologic response to neoadjuvant treatment in locally advanced rectal cancer and impact on outcome. J Gastrointest Oncol 7: 603-608, 2016.

13. Wasmuth HH, Rekstad LC and Tranø G: The outcome and the frequency of pathological complete response after neoadjuvant radiotherapy in curative resections for advanced rectal cancer: A population-based study. Colorectal Dis 18: 67-72, 2016.

14. Ponting CP, Oliver PL and Reik W: Evolution and functions of long noncoding RNAs. Cell 136: 629-641, 2009.

15. Han D, Wang M, Ma N, Xu Y, Jiang Y and Gao X: Long noncoding RNAs: Novel players in colorectal cancer. Cancer Lett 361: 13-21, 2015.

16. Ojima E, Inoue Y, Watanabe H, Hiro J, Toiyama Y, Miki C and Kusunoki M: The optimal schedule for 5-fluorouracil radiosensitization in colon cancer cell lines. Oncol Rep 16: 1085-1091, 2006.

17. Li YJ, Dong BK, Fan M and Jiang WX: BTG2 inhibits the proliferation and metastasis of osteosarcoma cells by suppressing the PI3K/AKT pathway. Int J Clin Exp Pathol 8: 12410-12418, 2015.

18. The Gene Ontology Consortium: Expansion of the gene ontology knowledgebase and resources. Nucleic Acids Res 45 (Database Issue): D331-D338, 2017.

19. The Gene Ontology Consortium, Ashburner M, Ball CA, Blake JA, Botstein D, Butler H, Cherry JM, Davis AP, Dolinski K, Dwight SS, et al: Gene ontology: Tool for the unification of biology. Nat Genet 25: 25-29, 2000. 
20. Kanehisa M, Furumichi M, Tanabe M, Sato Y and Morishima K KEGG: New perspectives on genomes, pathways, diseases and drugs. Nucleic Acids Res 45: D353-D361, 2017.

21. Kanehisa M and Goto S: KEGG: Kyoto encyclopedia of genes and genomes. Nucleic Acids Res 28: 27-30, 2000.

22. Kanehisa M, Sato Y, Kawashima M, Furumichi M and Tanabe M: KEGG as a reference resource for gene and protein annotation. Nucleic Acids Res 44: D457-D462, 2016.

23. Livak KJ and Schmittgen TD: Analysis of relative gene expression data using real-time quantitative PCR and the 2(-Delta Delta C(T)) method. Methods 25: 402-408, 2001.

24. Chen WM, Huang MD, Sun DP, Kong R, Xu TP, Xia R, Zhang EB and Shu YQ: Long intergenic non-coding RNA 00152 promotes tumor cell cycle progression by binding to EZH2 and repressing p15 and p21 in gastric cancer. Oncotarget 7: 9773-9787, 2016.

25. Li J, Yu H, Xi M and Lu X: Long noncoding RNA C17orf91 is a potential prognostic marker and functions as an oncogene in ovarian cancer. J Ovarian Res 9: 49, 2016.

26. Takahashi Y, Sawada G, Kurashige J, Uchi R, Matsumura T, Ueo H, Takano Y, Eguchi H, Sudo T, Sugimachi K, et al: Amplification of PVT-1 is involved in poor prognosis via apoptosis inhibition in colorectal cancers. Br J Cancer 110: 164-171, 2014.

27. Kawamoto A, Yokoe T, Tanaka K, Saigusa S, Toiyama Y, Yasuda H, Inoue Y, Miki C and Kusunoki M: Radiation induces epithelial-mesenchymal transition in colorectal cancer cells. Oncol Rep 27: 51-57, 2012.

28. Marcondes PGD, Bastos LG, Rocha MR and Morgado-Díaz JA: EphA4-mediated signaling regulates the aggressive phenotype of irradiation survivor colorectal cancer cells. Tumour Biol 37 : 12411-12422, 2016.

29. Tato-Costa J, Casimiro S, Pacheco T, Pires R, Fernandes A, Alho I, Pereira P, Costa P, Castelo HB, Ferreira J and Costa L: Therapy-induced cellular senescence induces epithelial-to-mesenchymal transition and increases invasiveness in rectal cancer. Clin Colorectal Cancer 15: 170-178, 2016.

30. Ke CL, Chen ZH, Shi JF, et al: X-ray irradiation on the expression of Kiss-1 gene and the potential of cell invasion and migration in colorectal cancer cell line HCT116. J Front Med: 14-15, 2014

31. Moncharmont C, Levy A, Guy JB, Falk AT, Guilbert M, Trone JC, Alphonse G, Gilormini M, Ardail D, Toillon RA, et al: Radiation-enhanced cell migration/invasion process: A review. Crit Rev Oncol Hematol 92: 133-142, 2014.

32. Yu Y, Yang J, Li Q, Xu B, Lian Y and Miao L: LINC00152: A pivotal oncogenic long non-coding RNA in human cancers. Cell Prolif 50, 2017

33. Zhao J, Liu Y, Zhang W, Zhou Z, Wu J, Cui P, Zhang Y and Huang G: Long non-coding RNA Linc00152 is involved in cell cycle arrest, apoptosis, epithelial to mesenchymal transition, cell migration and invasion in gastric cancer. Cell Cycle 14: 3112-3123, 2015.

34. Yu J, Liu Y, Guo C, Zhang S, Gong Z, Tang Y, Yang L, He Y, Lian Y,Li X, et al: Upregulated long non-coding RNA LINC00152 expression is associated with progression and poor prognosis of tongue squamous cell carcinoma. J Cancer 8: 523-530, 2017.
35. Wang Y, Liu J, Bai H, Dang Y, Lv P and Wu S: Long intergenic non-coding RNA 00152 promotes renal cell carcinoma progression by epigenetically suppressing P16 and negatively regulates miR-205. Am J Cancer Res 7: 312-322, 2017.

36. Feng S, Zhang J, Su W, Bai S, Xiao L, Chen X, Lin J, Reddy RM, Chang AC, Beer DG and Chen G: Overexpression of LINC00152 correlates with poor patient survival and knockdown impairs cell proliferation in lung cancer. Sci Rep 7: 2982, 2017.

37. Cai Q, Wang Z, Wang S, Weng M, Zhou D, Li C, Wang J, Chen E and Quan Z: Long non-coding RNA LINC00152 promotes gallbladder cancer metastasis and epithelial-mesenchymal transition by regulating HIF-1 $\alpha$ via miR-138. Open Biol 7: pii: 160247, 2017.

38. Zhang XL, Zhu Y, Li SN, Fan SQ, Yuan P, Gao XD, Wang CJ and Zhang FY: Expression of long non-coding RNA LINC00152 in human colon cancer and its clinical significance. Chin J Clin Laborat Sci: 354-358, 2015 (In Chinese).

39. Yue B, Cai D, Liu C, Fang C and Yan D: Linc00152 functions as a competing endogenous RNA to confer oxaliplatin resistance and holds prognostic values in colon cancer. Mol ther 24: 2064-2077, 2016.

40. Ji J, Tang J, Deng L, Xie Y, Jiang R, Li G and Sun B: LINC00152 promotes proliferation in hepatocellular carcinoma by targeting EpCAM via the mTOR signaling pathway. Oncotarget 6 : 42813-42824, 2015.

41. Chen QN, Chen X, Chen ZY, Nie FQ, Wei CC, Ma HW, Wan L, Yan S, Ren SN and Wang ZX: Long intergenic non-coding RNA 00152 promotes lung adenocarcinoma proliferation via interacting with EZH2 and repressing IL24 expression. Mol Cancer 16: 17, 2017.

42. Cai Q, Wang ZQ, Wang SH, Li C, Zhu ZG, Quan ZW and Zhang WJ: Upregulation of long non-coding RNA LINC00152 by SP1 contributes to gallbladder cancer cell growth and tumor metastasis via PI3K/AKT pathway. Am J Transl Res 8: 4068-4081, 2016

43. Qiu JJ and Yan JB: Long non-coding RNA LINC01296 is a potential prognostic biomarker in patients with colorectal cancer. Tumour Biol 36: 7175-7183, 2015.

44. Zhang YH,Fu J,Zhang ZJ, Ge CC and Yi Y: LncRNA-LINC00152 down-regulated by miR-376c-3p restricts viability and promotes apoptosis of colorectal cancer cells. Am J Transl Res 8: 5286-5297, 2016.

45. Tani $\mathrm{H}$ and Torimura $\mathrm{M}$ : Identification of short-lived long non-coding RNAs as surrogate indicators for chemical stress response. Biochem Biophys Res Commun 439: 547-551, 2013.

46. Vidyasekar P, Shyamsunder P, Arun R, Santhakumar R, Kapadia NK, Kumar R and Verma RS: Genome wide expression profiling of cancer cell lines cultured in microgravity reveals significant dysregulation of cell cycle and MicroRNA gene networks. PLoS One 10: e0135958, 2015.

This work is licensed under a Creative Commons Attribution-NonCommercial-NoDerivatives 4.0 International (CC BY-NC-ND 4.0) License. 